
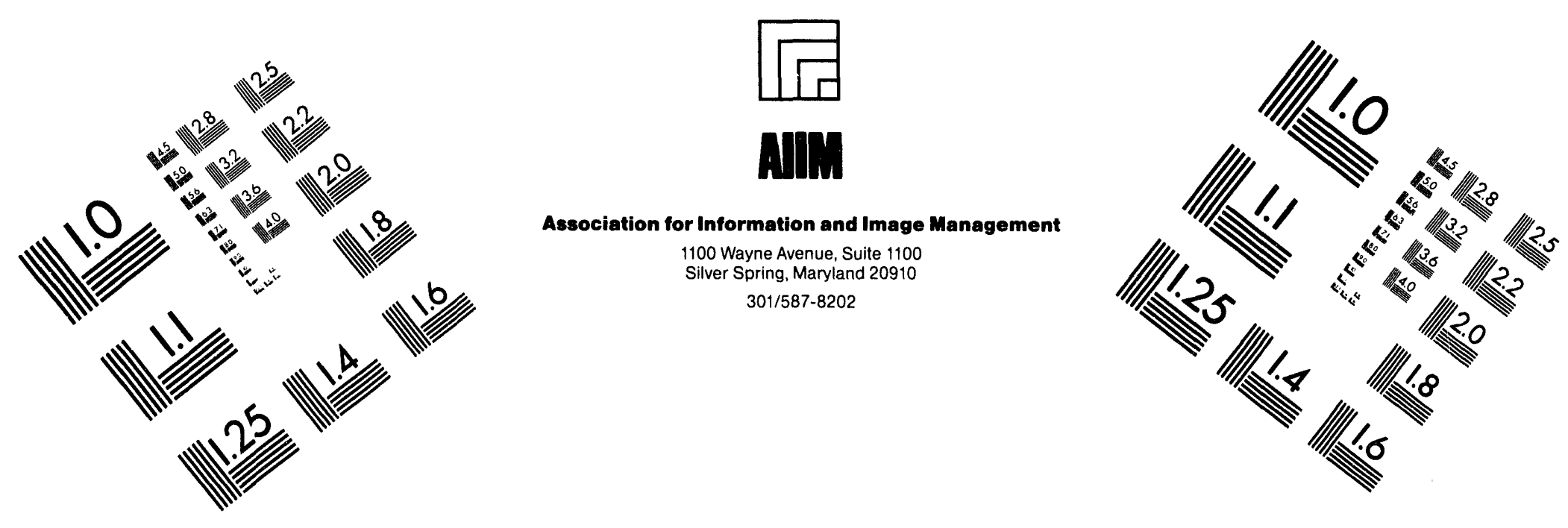

Centimeter

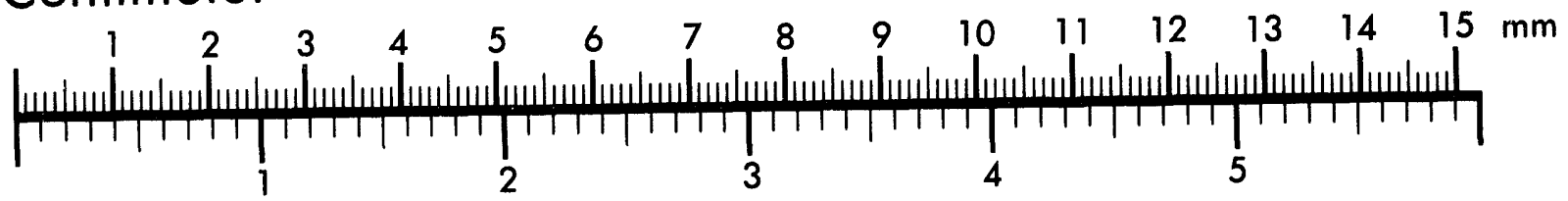
Inches
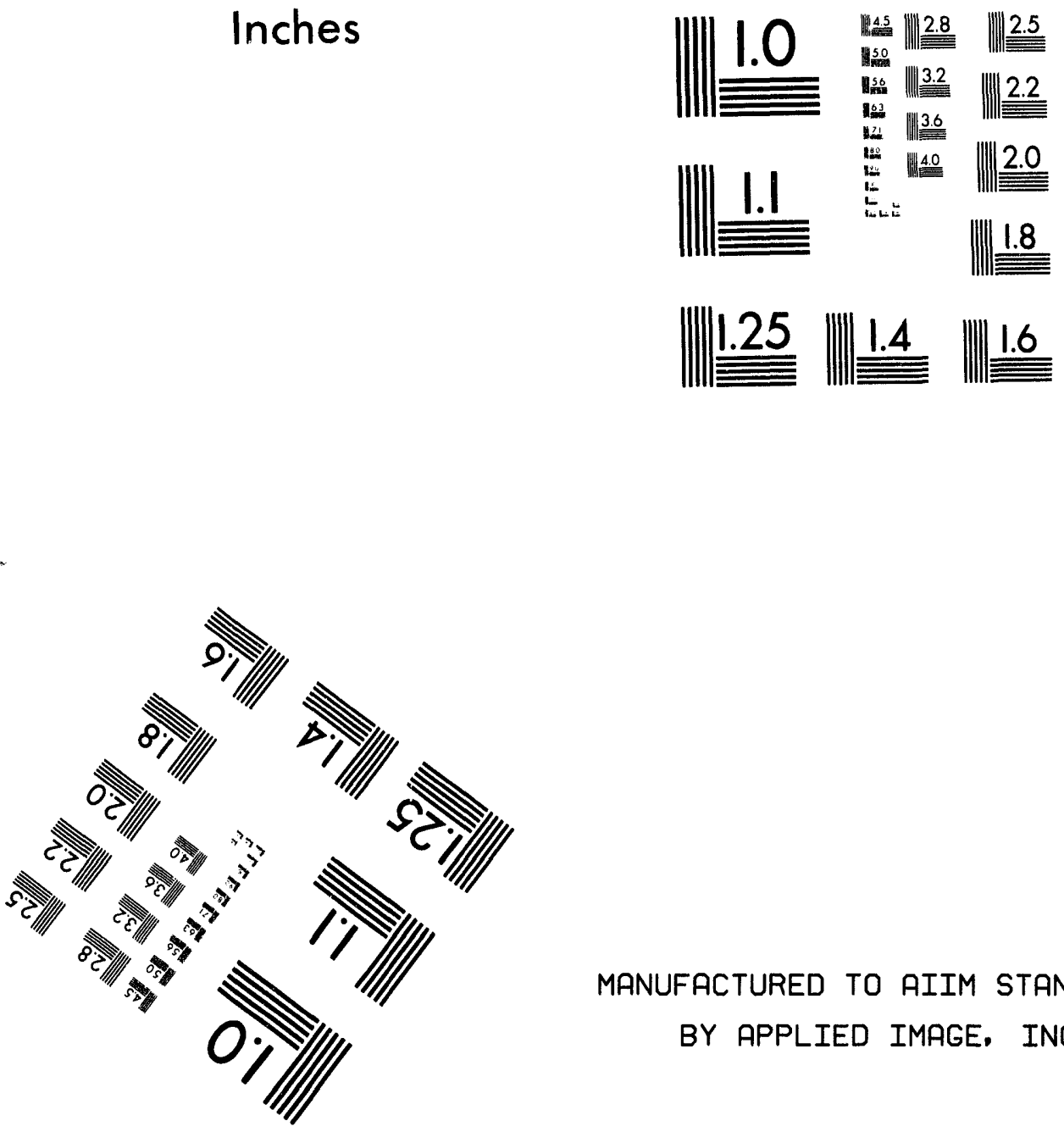

MANUFACTURED TO AIIM STANDARDS

BY APPLIED IMAGE. INC.

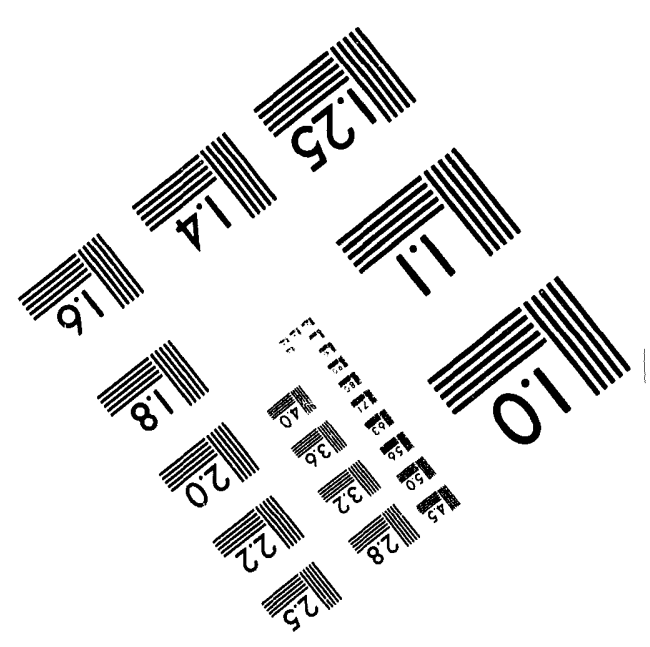



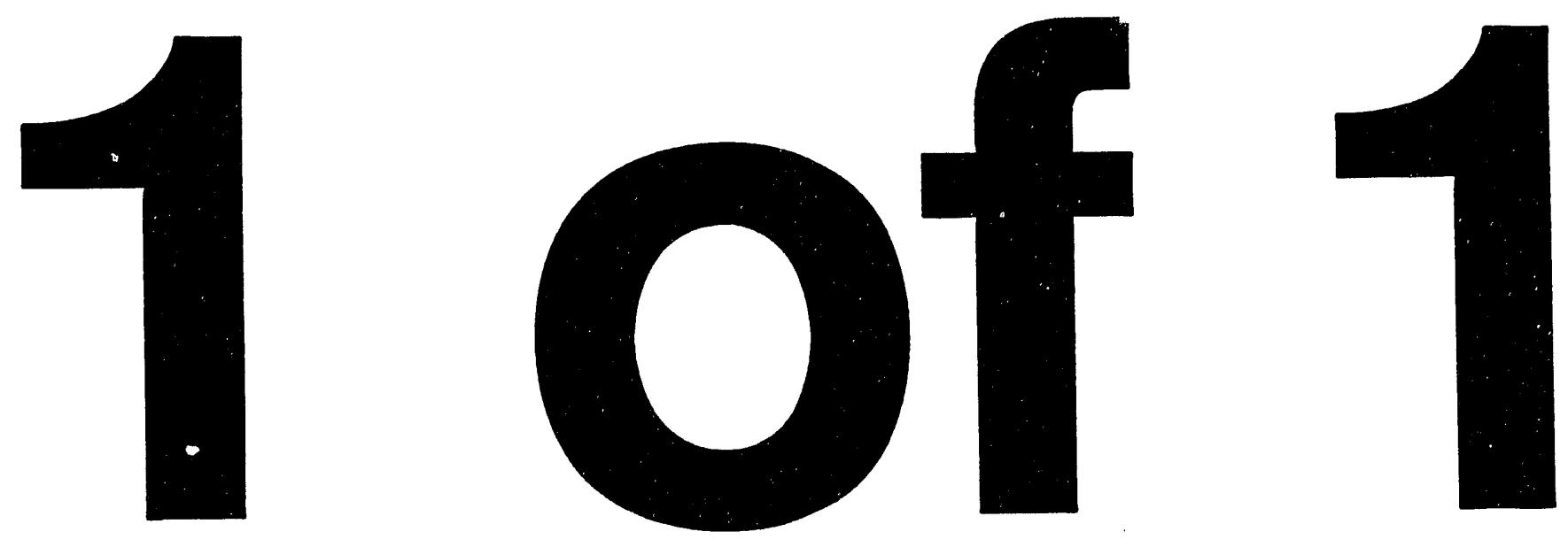
Submitted to 13th International Conference

on Magnet Technology, Victoria B.C., 9/20-24/93

BNL -49501

\title{
Shimming Techniques for the Ultraprecise Muon g-2 Storage Ring at the AGS*
}

\author{
G.T.Danby and J.W. Jackson \\ Alternating Gradient Synchrotron, Brookhaven National Laboratory \\ Upton, New York 11973
}

\begin{abstract}
Major components are in an advanced state of construction for a 7 meter radius 0.1 PPM precision storage ring. ${ }^{1}$ Detailed techniques are planned for static shimming of the assembly to at least 10 PPM magnetic field uniformity prior to the use of field correction coils. An air gap behind each ultra-pure iron pole piece strongly decouples the aperture field shape from the properties of the $\mathbf{1 0 0 6}$ iron yoke. Iron wedges whose thickness varies across the width of the poles with slope of $\sim 1 / 60$ are used to eliminate the gradient produced by the $\mathrm{C}$-magnet shape required for open access for the decay electron counter on the inside radius of the storage ring magnet. These wedges are $10 \mathrm{~cm}$ in azimuthal length and can be radially adjusted for short wavelength field adjustments. A horizontal motion of $50 \mu \mathrm{m}$ effectively adjusts the $10 \mathrm{~cm}$ half-gap aperture by $1 \mu \mathrm{m}$ (or 10 PPM). This and other techniques to adjust dipole, quadrupole, sextuple, etc. multipoles will be described.
\end{abstract}

\section{INTRODUCTION}

An ultraprecise storage ring is being constructed which, in conjunction with the very high intensity of pion and muon beams now available from the AGS with its new Booster, should permit measurement of $A \mu=(g-2) / 2$ to 0.35 PPM for the muon. The experiment will observe the spin motion of polarized muons in a uniform magnetic field B. The spin precession angular frequency Ws is greater than the cyclotron angular frequency Wc. The $g-2$ precession frequency $\mathrm{Wa}=\mathrm{Ws}-\mathrm{Wc}=\mathrm{e} / \mathrm{mc}_{\mathrm{A}} \mathrm{A}_{\mu} \mathrm{B}$. Now $\mathrm{e} / \mathrm{mc}$ is known; Wa and integral $B$ must be measured. The goal is a factor of 20 improvement over a previous similar experiment. ${ }^{2,3}$ This measured $A \mu$ to 7 PPM.

Vertical focusing is provided by electrostatic quadrupoles distributed around most of the storage ring. For a "magic" momentum $\mathrm{P} \mu=3.09 \mathrm{GeV} / \mathrm{c}$, or $\gamma=29.3$, the electric field has no effect on the precessional frequency, ${ }^{3}$

$$
W a=\frac{e}{m c}\left[A_{\mu} B-\left(A_{\mu}-\frac{1}{\gamma^{2}-1}\right)|B x E|\right] .
$$

The field B must be made very uniform to that its integral around the storage ring azimuth and over the ring

\footnotetext{
- Work performed under the auspices of the U.S. Dept. of Energy Contract No. DE-AC02-76CH0OO16.
}

acceptance orbits can be known to 0.1 PPM, less than the expected statistical error of 0.3 PPM.

Figure 1 shows a plan view. Either pion or direct muon injection will be used. Pions will enter the storage ring through an inflector which cancels the storage ring field until the pions are very close to the stored beam acceptance. Longitudinally polarized muons produced by the decay of pions of slightly higher momentum are captured in the ring. For the direct muon injection option, a fast kicker is required to deflect the muons into the acceptance. The storage ring is an iron-dominated open Cmagnet. This permits electrons from muon decay to impinge on detectors distributed all around the inside radius. The electron direction is correlated with muon spin direction, so muon decay is modulated by Wa.

The muon $\mathrm{g}-2$ is a major experiment, now in an advanced state of final design and construction, with many features which have been described in detail. ${ }^{4,5} \mathrm{~A}$ major challenge of the experiment is the very high accuracy required. The authors have been working for a decade on the storage ring magnet, ${ }^{6}$ both to produce a workable economic design and on means to shim it to high precision once operating. Part of this work is the subject of this paper.

\section{THE STORAGE RING MAGNET}

The storage ring uses an iron dominated " $14.5 \mathrm{kG}$ superferric" magnet which approximates as closely as practical a continuous ring. ${ }^{1}$ The magnetic field would only be $10 \%$ with the superconducting ring coils alone (no $\mathrm{Fe}$ ). Figures 2, 3, and 4 show the cross section. The Cmagnet yoke, which has been fabricated from conventional 1006 steel, is the dominant cost factor. Economic viability dictated minimizing its size, weight and cost. The air gap between the yoke and each pole strongly decouples the detailed field shape in the storage region from the yoke, except for the basic reluctance and dipolar field amplitude. The pole pieces are being fabricated from very pure vacuum cast iron ( $<$ 10005). This not only gives improved permeability, but minimizes variations in properties due to inclusions and voids. The poles have been carefully shaped to minimize the total flux while providing a very uniform field "on paper" over the acceptance of $p=4.5 \mathrm{~cm}$. The air gap of $1 \mathrm{~cm}$ at $\mathrm{r}=$ $711.2 \mathrm{~cm}$ adds $10 \%$ to the ampere turns required to excite the $9 \mathrm{~cm}$ half gap. The air gap tilts by $\pm 0.45 \mathrm{~cm}$ over \pm $27 \mathrm{~cm}$ of width. The smaller air gap on the inside (Fig. 4) 
compensates for all the flux returning on the outside, to eliminate a large $\mathrm{C}$-magnet gradient.

The two outer radius coils, which share a common cryostat basically excite $20 \mathrm{~cm}$ of $14.5 \mathrm{kG}$ field. The two inner coils serve to restrict the magnetomative force to the polar region. They are located beyond the air gaps vertically to provide access for shimming and to prevent a large negative hoop stress on the inner coils. The superconductor is heavily stabilized by co-extruded pure aluminum. Coil absolute positional tolerances are not critical. Forces and magnetic field imposed on the coil are modest. However, the very large diameter to crosssectional area ratio makes the design demanding. Superconducting coils permit a continuous ring magnet without interruptions for cooling water and the heat and vibration assuciated with conventional coils. Current ripple is greatly reduced and large power savings occur which in principle at least allows for very long runs of the storage ring without recycling.

The completed precision field design carefully optimized the field uniformity (Fig. 5). Both geometrical and material variations will cause deviations. Furthermore we do not believe the calculations to this accuracy. However great effort was put into computing perturbations and into allowing for adjustment of a continuous ring magnet after complete preliminary storage ring field mapping. The pole edge shims and air gap shims are adjustable. The field errors are expected to be of low multipoles within the range of corrections. The pole surfaces may have to be ground or otherwise locally adjusted if small range variations are sufficient to complicate the expected "on-line" mapping with a movable trolley inside the vacuum chamber.

\section{METHODS OF SHIMMMING}

The magnet is a bolted mechanical assembly. Tolerances could allow up to $0.2 \mathrm{~mm}\left(.008^{\prime \prime}\right)$ variation out of $20 \mathrm{~cm}$ of air gap, or 1000 PPM gap change and field change. Moving the poles up or down has a very weak effect. Poles will be accurately spaced and yoke tolerances will appear as changes in the shimming air gaps only. Shimming wedges are shown conceptually in Figs. 6 and 7. Adjustment in thickness, or radial adjustment for fine control, will be used to get the aperture centerline field $B_{0}$ uniform to 10 PPM or possibly better. Surface coils, which are being designed by others, will provide the last stage of static as well as dynamic control. Note that the wedge angle determines the quadrupole correction, so radial adjustment affects only dipole. The wedges will originally be oversize so that one stage of grinding is assumed after initial mapping. Reluctance adjusting shims on the top and bottom of the yoke also permit more remote adjustment. Major peturbations, notably the inflector, will require large iron shimming on the yoke in the immediate vicinity of the perturbation.
Sextupole, octupole and 10-pole aberrations are controlled by the pole edge shims, which can be removed for grinding and which will start off oversized prior to mapping. Table 1 illustrates this control. Symmetric variation (Column I) gives both sextupole and 10-pole. The shims will be set to eliminate 10-pole and the small residual sextupole will be well within the range of dynamic surface coil sextupole correction. Asymmetric shims (Column II) produce quadrupole, plus $41 \%$ octupole. This will be adjusted for zero octupole. Residual quadrupole will be removed by the air gap wedges angle (Column III). Table 1 also shows that with pole edge shims ground and replaced to an expected $.001^{\prime \prime}$ tolerance, the RMS errors due to edge shims for allowed and skew (not shown) multipoles will be four times smaller than the values shown in Columns I and II.

A $30 \mu \mathrm{m}$ radial displacement of all air gap wedges makes $0.5 \mu \mathrm{m}$ change in air gap, or 5 PPM change in dipole field. If only a single wedge pair was moved the effect locally is $\sim 1 / 7$ of the movement of a large number of wedges. The full width at half maximum of the field change of a single wedge pair is $0.5 \mathrm{~m}$ azimuthally, which overlaps two $10 \mathrm{~cm}$ wedges on each side of the displaced pair. Thus the number of wedges could be reduced to 3 or 4 rather than 12 per $10^{\circ}$ pole plate section. However, they would be required to be polar and no longer have parallel sides $10 \mathrm{~cm}$ apart. Wide wedges could not be pulled out to the inside radius.

\section{SPACES BETWEEN POLE SECTIONS}

Although an attempt was made to make the ring azimuthally continuous, in fact the flux return yoke is made of $30^{\circ}$ sections (see Fig. 1) which are butted up or shimmed to have minimum cracks between sections. Furthermore, each $30^{\circ}$ magnet segment has three $10^{\circ}$ pole piece sections which are fabricated with minimum space tolerance for (insulated) cracks between sections. These pole piece cracks will have both static and dynamic implications.

Figure 8 shows the computed vertical field depression due to a $50 \mu \mathrm{m}$ total space $( \pm 1 \mathrm{mil})$ between adjacent pole pieces, symmetrically on both top and bottom poles. $\mathrm{x}$ is approximately the azimuthal or beam direction and $\mathrm{x}$ $=0$ is directly between the spaces. On the horizontal midplane $(y=0)$ at $x=0$, the field is low by 13 gauss or 9 PPM. At $y= \pm 4.5 \mathrm{~cm}$, the maximum vertical acceptance, the field will be low by twice this amount at $x=0$, as shown in Fig. 8.

This $50 \mu \mathrm{m}$ space is in effect a dipolar line source normal to the beam of opposite sign to the magnet field. Integration of the field depression at $y=0$ over the azimuth gives 0.77 gauss-cm $\times 2$ (for minus $\times$ side) $=1.54$ gauss-cm. Since there are 36 pole plate pairs, the azimuthal integral is 55 gauss $-\mathrm{cm}$, or -0.86 PPM of the 
azimuthally integrated field. However, the azimuthal integral of the vertical field at $y=0,2,4$, and $5 \mathrm{~cm}$ all agree to $1 \%$.

Thus the effect of a small symmetric space between pole pieces, top and bottom, will complicate NMR magnetic measurement particularly at large y values, but not cause significant effect on the azimuthal integrals over the orbits. However, asymmetric spacings, such as the top spacing larger than the bottom spacing are a very different situation. This will be studied in the near future.

Since the pole faces are at $y= \pm 9 \mathrm{~cm}$, calculations show that a dipolar line current loop of, for example, $2 \mathrm{~cm}$ total width centered on the pole end spacings could be used to compensate for spacings. Whether this is useful is yet to be determined. Many actual imperfections in the poles, etc., can produce field variations comparable or larger than $50 \mu \mathrm{m}$ spacings between pole pieces.

The magnet will have the minimum spaces practical. The poles are attached to the yokes, which are attached to each other, so that uniform thermal expansion should change the radius slightly, not the pole spacings. When actual measurements are taken on the ring, whether systematic effects of pole spacings can be separated from other field error sources will be determined, and whether this information can be put to use to aid in field analysis or corrections.

Dynamic effects due to magnet charging, and especially possible magnet quenches, inducing eddy currents in indvidual magnet sections violate the continuous storage ring approximation. These are under study at the present time.

\section{REFERENCES}

[1] Brown et al.,"An ultra-precise storage ring for the muon $\mathrm{g}-2$ measurement," MT-11, Japan 1989.

[2] Final Report of the CERN Muon Storage Ring. J. Bailey et al. Nuclear Physics B150 (1979) 1-75.

[3] H. Drumm, "The Storage Ring Magnet of the Third Muon (g-2) Experiment at CERN" NMM 158, pp. 347-362 (1979).

[4] AGS Proposal. A New Precision Measurement of the Muon g-2 Value at the Level of 0.35 PPM. V.W. Hughes et al. Sept. 1985.

[5] Muon g-2 Design Report, AGS Exp. 821, July 1992.

[6] G.T. Danby and J.W. Jackson "Ultra precise magnet design and shimming studies" Internal g-2 Note 001,1987.
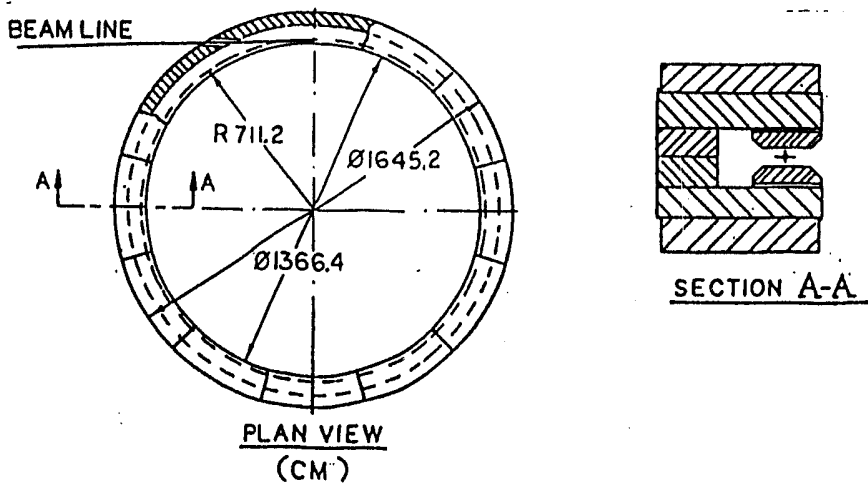

TABLE I

MULTIPOLES COMPUTED AT $\rho=4.5 \mathrm{~cm}$ For 3 PERTURBATIONS

\begin{tabular}{|c|c|c|c|}
\hline $\begin{array}{c}\text { Multipoles } \\
\text { Moment }\end{array}$ & $\begin{array}{c}\text { I } \\
\text { PPM }\end{array}$ & $\begin{array}{c}\text { II } \\
\text { PPM }\end{array}$ & $\begin{array}{c}\text { II } \\
\text { PPM }\end{array}$ \\
\hline (Quad 1) & -.2 & +9.2 & +28.80 \\
\hline (Sext) 2 & +7.5 & 0 & +2.80 \\
\hline 3 & -0.1 & +3.8 & 0 \\
\hline 4 & +1.4 & 0 & +0.10 \\
\hline 5 & 0 & +0.4 & -0.05 \\
\hline 6 & +.1 & 0 & -0.05 \\
\hline 7 & 0 & 0 & 0 \\
\hline 8 & 0 & 0 & -0.05 \\
\hline
\end{tabular}

Column I All four pole edge bumps - $50 \mu \mathrm{m}$ thicker.

Column II Small $\mathrm{r}$ bumps $50 \mu \mathrm{m}$ thinner, large $\mathrm{r}$ bumps $50 \mu \mathrm{m}$ Column III Increase pole base air gap wedge by 27 millirads, 20
times fabrication tolerances.

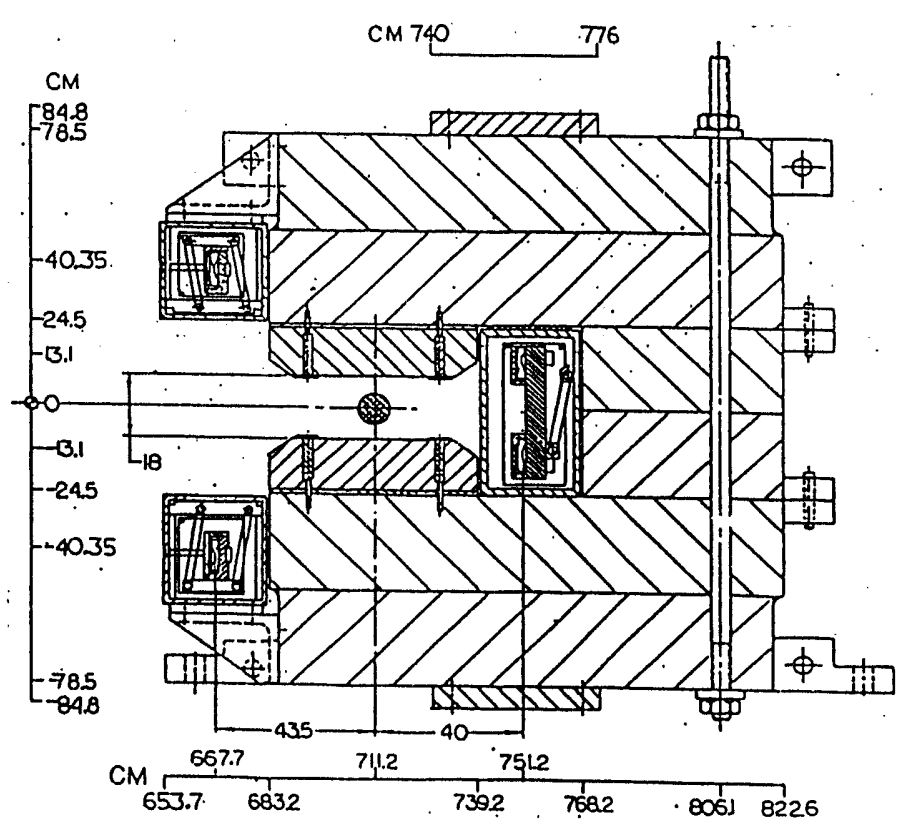

Fig. 2. g-2 magnet cross section.

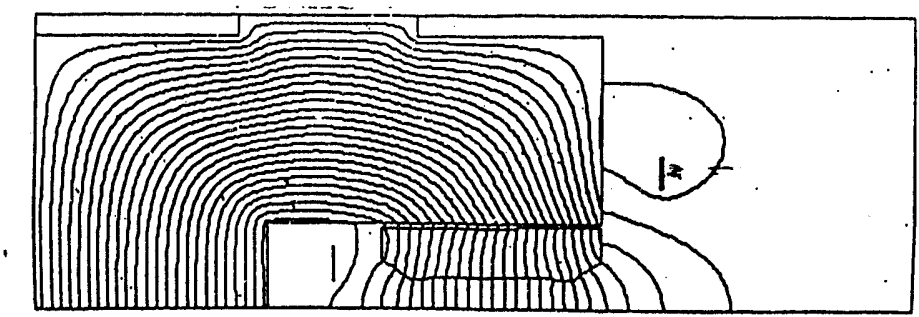

Fig. 3. Magnet flux pattern: 


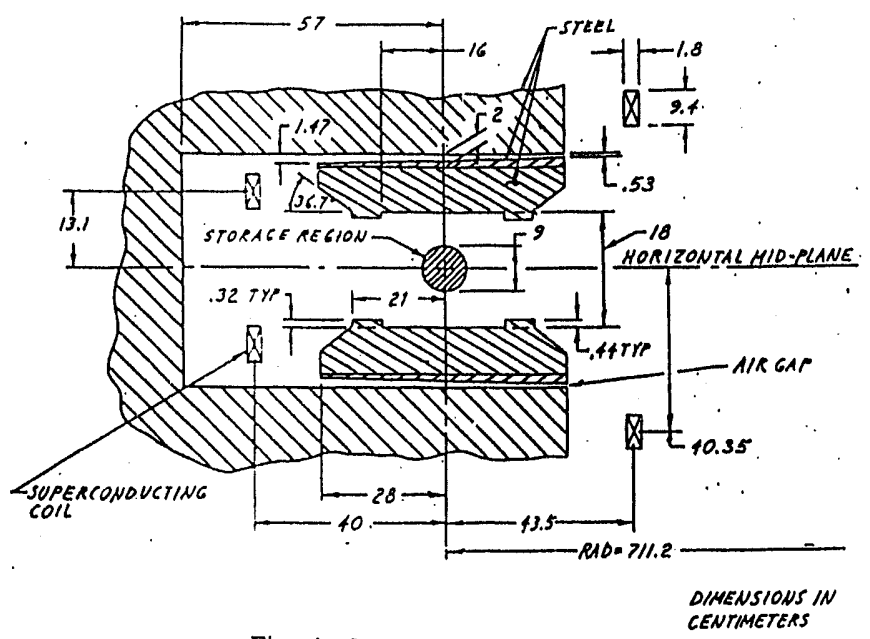

Fig. 4. Magnet pole region.

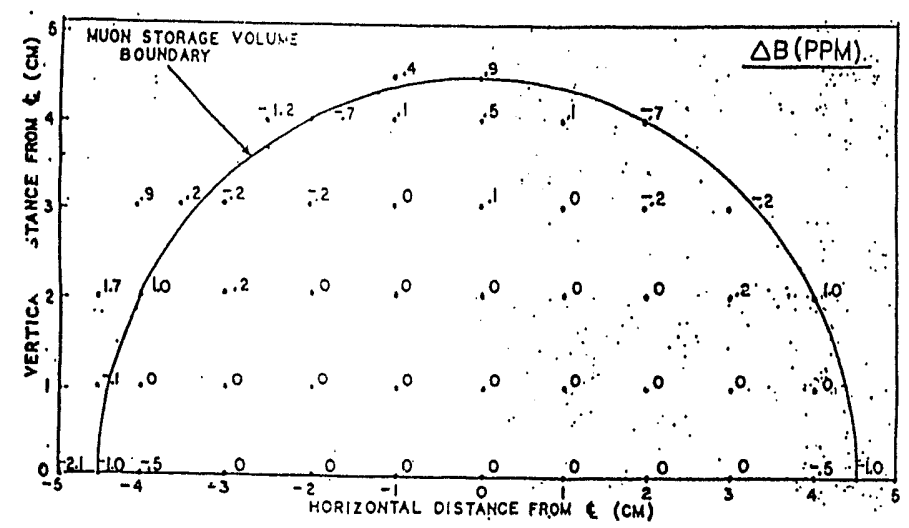

Fig. 5. Storage region field uniformity.

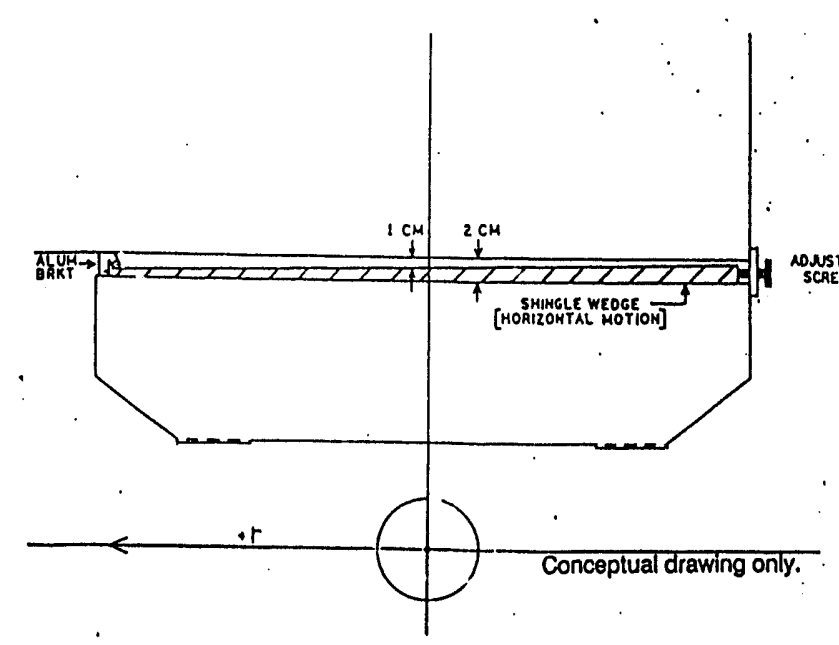

Fig. 6. Pole cross section showing wedge.

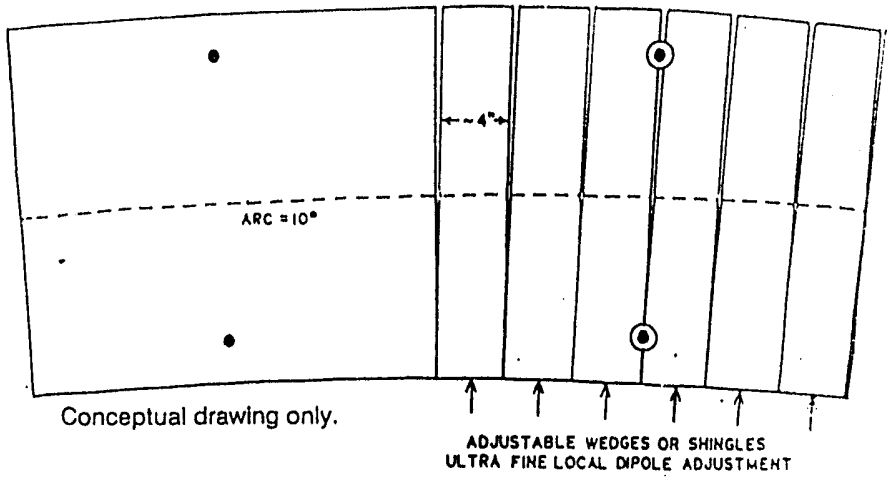

Fig. 7. Pole piece plus wedges in air gap.

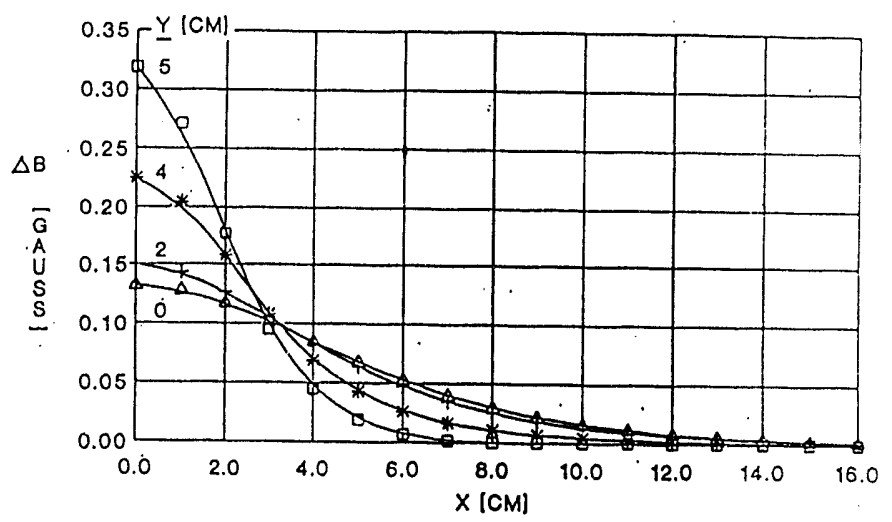

Fig. 8. Field depression due to 2 mil space between pole pieces.

\section{DISCLAIMER}

This report was prepared as an account of work sponsored by an agency of the United States Government. Neither the United States Government nor any agency thereof, nor any of their employees, makes any warranty, express or implied, or assumes any legal liability or responsibility for the accuracy, completeness, or usefulness of any information, apparatus, product, or process disclosed, or represents that its use would not infringe privately owned rights. Reference herein to any specific commercial product, process, or service by trade name, trademark, manufacturer, or otherwise does not necessarily constitute or imply its endorsement, recommendation, or favoring by the United States Government or any agency thereof. The views and opinions of authors expressed herein do not necessarily state or reflect those of the United States Government or any agency thereof. 

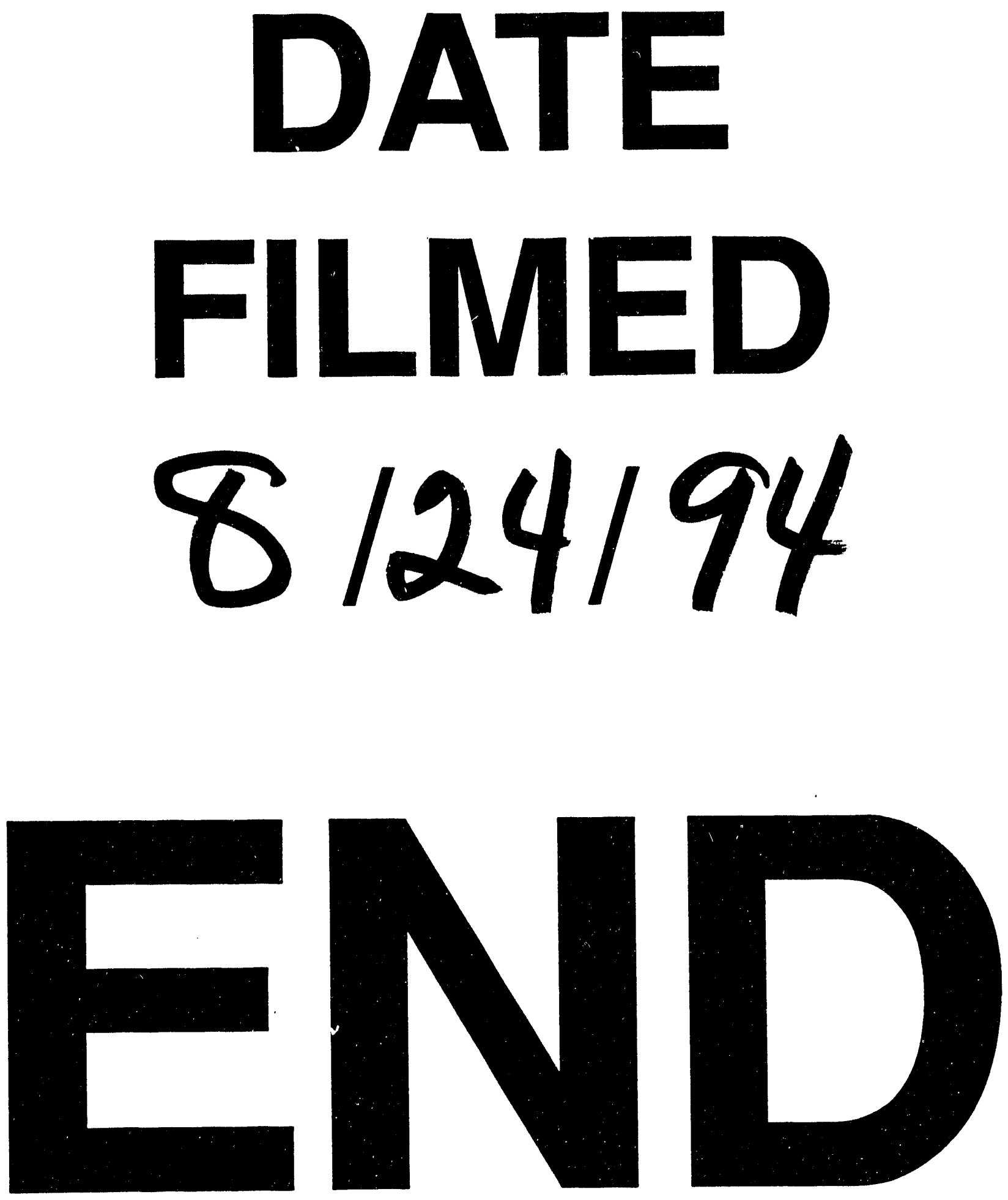
